\title{
The L dwarf/T dwarf transition: multiplicity, magnetic activity and mineral meteorology across the hydrogen burning limit.
}

\author{
Adam J. Burgasser ${ }^{1, \star}$ \\ University of California San Diego, MC 0424, 9500 Gilman Drive, La Jolla, CA 92093, USA \\ Received XXXX, accepted XXXX \\ Published online XXXX
}

Key words infrared: stars, stars: binaries: spectroscopic, stars: low-mass, brown dwarfs, techniques: spectroscopic

The transition between the $\mathrm{L}$ dwarf and $\mathrm{T}$ dwarf spectral classes is one of the most remarkable along the stellar/brown dwarf Main Sequence, separating sources with photospheres containing mineral condensate clouds from those containing methane and ammonia gases. Unusual characteristics of this transition include a $1 \mu \mathrm{m}$ brightening between late $\mathrm{L}$ and early T dwarfs observed in both parallax samples and coeval binaries; a spike in the multiplicity fraction; evidence of increased photometric variability, possibly arising from patchy cloud structures; and a delayed transition for young, planetary-mass objects. All of these features can be explained if this transition is governed by the "rapid" (nonequlibrium) rainout of clouds from the photosphere, triggered by temperature, surface gravity, metallicity and (perhaps) rotational effects. While the underlying mechanism of this rainout remains under debate, the transition is now being exploited to discover and precisely characterize tight $(<1 \mathrm{AU})$ very low-mass binaries that can be used to test brown dwarf evolutionary and atmospheric theories, and resolved binaries that further constrain the properties of this remarkable transition.

Copyright line will be provided by the publisher

\section{Introduction}

The $\mathrm{L}$ dwarf and $\mathrm{T}$ dwarf spectral classes encompass what were until recently the lowest-temperature, lowest mass (VLM) stars and brown dwarfs known, with $T_{\text {eff }} \approx 600-2200 \mathrm{~K}$ (see contribution by J. D. Kirkpatrick on the newly-defined Y dwarf class). While these classification classes are defined at different wavelengths (Kirkpatrick et al. 1999; Burgasser et al. 2006a), they are readily distinguished by their infrared (IR) spectra, dominated by $\mathrm{H}_{2} \mathrm{O}, \mathrm{CO}, \mathrm{FeH}$, and alkali absorption for the $\mathrm{L}$ dwarfs and $\mathrm{H}_{2}, \mathrm{H}_{2} \mathrm{O}, \mathrm{CH}_{4}$, and $\mathrm{NH}_{3}$ absorption for the $\mathrm{T}$ dwarfs. The IR spectral energy distributions (SEDs) of these classes are also distinct, $\mathrm{L}$ dwarfs being quite red in near-IR colors $(J-K \sim 1.5-2.5)$ and $\mathrm{T}$ dwarfs being relatively blue $(J-K \lesssim 1)$. This variation is due in large part to condensate cloud opacity, which arises as $\mathrm{TiO}, \mathrm{VO}, \mathrm{Fe}$ and other metals condense out of the atmosphere to form 10-30 $\mu \mathrm{m}$ grains in the photosphere (Ackerman \& Marley 2001; Helling et al. 2008). These grains scatter efficiently across the near-IR, having their greatest influence in the $1.0-1.3 \mu \mathrm{m}$ gas opacity window and giving rise to the red colors and muted $\mathrm{H}_{2} \mathrm{O}$ bands in the near-IR spectral of these objects (Allard et al. 2001). There are also pronounced variations in $\mathrm{L}$ and $\mathrm{T}$ dwarf SEDs that correlate with differences in surface gravity, metallicity, condensate cloud properties, and nonequilibrium chemistry (Looper et al. 2008; Burgasser et al. 2008; Cushing et al. 2010).

^ Corresponding author: e-mail: aburgasser@ucsd.edu

\section{Evolution across the $\mathrm{L} / \mathrm{T}$ transition}

Given their distinct spectral morphologies, and the fact that all brown dwarfs evolve through the $\mathrm{L}$ and $\mathrm{T}$ spectral sequence as they cool, the transition between these classesdefined here as spectral types L8 to T5-is key to understanding the atmospheric chemistry, thermal evolution and statistical distribution of brown dwarfs in the Galaxy. Formally, the start of the $\mathrm{T}$ dwarf class is defined by the emergence of the $1.6 \mu \mathrm{m} \mathrm{CH}_{4}$ band, but the observed changes in SED across the L/T transition also reflects a sudden removal of condensate grain opacity from brown dwarf photospheres over a relatively narrow $T_{\text {eff }}$ range. The rapidity of this shift is evident in the brightening of early-type T dwarfs around $1 \mu \mathrm{m}$-the so-called "J-band bump"-that is observed in both parallax samples (Tinney et al. 2003; Vrba et al. 2004) and between components of binaries spanning the transition (Burgasser et al. 2006b; Liu et al. 2006; Looper et al. 2008; see below). As opacity in this region is dominated by condensates in the L dwarfs, the observed brightening, along with the resurgence of $\mathrm{FeH}$ and alkali line absorption in the $1 \mu \mathrm{m}$ region (Burgasser et al. 2002), indicates a loss of condensates rather than obscuration from emergent $\mathrm{CH}_{4}$ absorption. A relatively sharp transition is also suggested by the small change in luminosity that encompasses the transition (Golimowski et al. 2004), the rarity of early-type $\mathrm{T}$ dwarfs in current search samples (Metchev et al. 2008), and an apparent excess of binaries at the $\mathrm{L} / \mathrm{T}$ transition (Burgasser 2007a). These lines of evidence suggest that the $\mathrm{L} / \mathrm{T}$ transition may be a relatively short-lived phase of brown dwarf evolution, although consideration must be taken into 

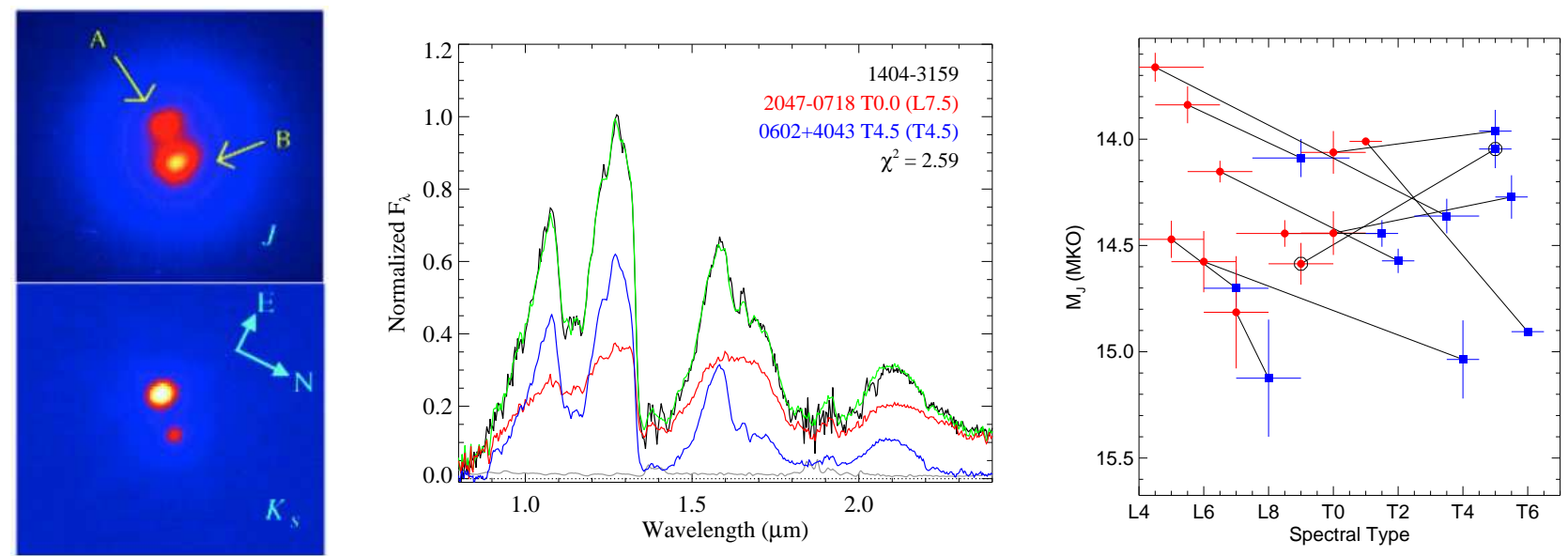

Fig. 1 Left: Adaptive optics images of the L/T transition binary 2MASS J1404-3159AB at $J$ (top) and $K_{s}$ (bottom). Primary (A) and secondary (B) are labeled, and are designated based on their $K_{s}$-band flux (from Looper et al. 2008). Center: Combined-light near-infrared spectrum of 2MASS J1404-3159AB (black line) compared to the best fit template binary (green or medium grey line) composed of a T0 primary (red or dark grey line) and a T4.5 secondary (blue or lower light grey line), based on resolved photometry. The secondary is considerably brighter than the primary at $1.05 \mu \mathrm{m}$ and $1.27 \mu \mathrm{m}$ (from Burgasser et al. 2010). Right: Absolute MKO J-band magnitudes of resolved L/T transition binaries with measured parallaxes as a function of component type, based on the compilation of Dupuy \& Liu (2012). Primaries are indicated by red (or light grey) circles, secondaries by blue (or dark grey) squares, and pairs are joined. Lines that slope upward, including 2MASS J1404-3159AB (large black circles), are flux reversal binaries.

account for possible feedback in the thermal evolution at this phase (Saumon \& Marley 2008).

Static 1D atmosphere models for brown dwarfs which parameterize condensate cloud properties (e.g., $f_{\text {sed }}$ in Ackerman \& Marley 2001) predict a much slower decline in condensate opacity with $T_{e f f}$ as cloud tops sink to deeper layers. (Marley et al. 2002). To reproduce the transition, a change in cloud surface coverage or condensate grain properties must be introduced. The first possibility, inspired by the patchy tropospheric clouds of Jupiter and Saturn, predicts that holes in the cloud layer form at the L/T transition, allowing "hotter" light to emerge from below (Ackerman \& Marley 2001; Burgasser et al. 2002; Marley et al. 2010). The second possibility, a global change in the cloud thickness at the L/T transition, may reflect a "rainout" of the upper cloud decks by dynamic perturbations (Knapp et al. 2004; Saumon \& Marley 2008). Either of these cases may be driven by changes in the convective structure of the photosphere; properly tuned, both can reproduce the $J$-band brightening and changes to brown dwarf SEDs (Cushing et al. 2008)

A potential discriminator of these hypotheses is the possibility that cloud fragmentation could induce more frequent and intense variability at the $\mathrm{L} / \mathrm{T}$ transition, particularly aroun $1 \mu \mathrm{m}$, as cool clouds and hot holes rotate in and out of view. The recent detection of two strongly variable brown dwarfs, SIMP J0136+0933 (Artigau et al. 2009) and 2MASS J2139+0220

(Radigan et al. 2012), both of which are early-type T dwarfs, are seen to support this idea. However, there is currently limited theoretical work on cloud structure in rapidly rotating brown dwarfs to make a robust prediction of this effect
(Schubert \& Zhang 2000; Sanchez-Lavega 2001), and variability samples are currently too sparse to robustly identify trends. As both $\mathrm{H} \alpha$ and radio emission have been detected in cool T dwarfs (Route \& Wolszczan 2012), magnetic spots or auroral emission cannot be ruled out as a source for this variability (Lane et al. 2007; Berger et al. 2009).

\section{Binaries and the $L / T$ transition}

\subsection{Resolved binaries: tracing excess}

One of the key lines of evidence that condensates are removed from the photospheres of $\mathrm{L} / \mathrm{T}$ transition objects is the $1 \mu \mathrm{m}$ brightening observed between "flux reversal" binaries that straddle this transition. Figure 1 shows an example of one such source, 2MASS J1404-3159AB (Looper et al. 2008), whose T4.5 secondary is $\sim 0.5$ mag brighter at $J$ and $\sim 1$ mag fainter at $K$ than its T0 primary. The excess is concentrated in the $1.05 \mu \mathrm{m}$ and $1.27 \mu \mathrm{m}$ flux peaks, the regions most affected by condensate opacity in the $\mathrm{L}$ dwarfs. However, not all binaries that straddle the $\mathrm{L} / \mathrm{T}$ transition are flux-reversal systems; there is in fact considerable variation in the absolute brightnesses and relative flux trends among these systems, suggesting that additional secondary parameters play a role in governing when and how quickly brown dwarfs evolve across the L/T transition (see below).

\subsection{Spectral binaries: probing small separations}

The dramatic SED changes that occur across the L/T transition also facilitate the identification of spectral binaries, 
or blended-light pairs. One of the first L/T binaries discovered, 2MASS J0518-2828AB, was initially recognized as a peculiar source whose near-infrared spectrum could be reproduced as a late $\mathrm{L}$ dwarf and mid-type $\mathrm{T}$ dwarf composite (Cruz et al. 2004). Since then, nearly two dozen spectral binaries and binary candidates have been identified (Burgasser 2007b; Burgasser et al. 2008a, 2010, 2011, 2012; Gelino \& Burgasser 2010; Kirkpatrick et al. 2010; Gei $\beta$ ler et al. 2011). Some have been resolved, allowing for robust assessments of their component SEDs, orbital characteristics and physical properties. However, spectral binaries also allow us to identify systems that are too tightly separated or too distant to be resolved by direct imaging. This is relevant to our understanding brown dwarf formation, as the incidence of tight brown dwarf binaries ( $\rho<1 \mathrm{AU})$, and hence the overall binary fraction, remains poorly constrained (330\%; Basri \& Reiners 2006; Joergens 2008; Blake et al. 2010). Identification of these intrinsically faint systems through radial velocity $(\mathrm{RV})$ monitoring and microlensing programs remains inefficient. Yet two of the four known VLM binaries with $\rho<0.5$ AU 2MASS J0320-0446AB (Burgasser et al. 2008; Blake et al. 2008) and SDSS J0006-0852AB (Burgasser et al. 2012), were both identified as spectral binaries. By combining the component $T_{e f f} \mathrm{~s}$ of these systems (from spectral binary analysis) with their system mass function (from the RV orbit), one obtains constraints on the age and orbital inclination of these systems based on evolutionary models (Fig. 2). Where independent age information is available, the evolutionary models themselves can be tested 11 Tight spectral binaries therefore complement studies of astrometric binaries in testing brown dwarf theory (Dupuy et al. 2009; Konopacky et al. 2010), but on considerably shorter timescales.

Not all blended-light systems will turn out to be physical binaries. A case in point is the photometrically variable T2.5 dwarf 2MASS J2139+0220, which was identified as a spectral binary (Burgasser et al. 2010), but is unresolved in HST imaging (Radigan et al. 2012) and does not appear to be an RV variable (Khandrika et al. 2012). The spectral binary nature of this source may instead reflect the distinct SEDs emerging from cloudy and non-cloudy regions on its surface, and may suggests a method of finding other highly variable sources.

\section{Parameters governing the $\mathrm{L} / \mathrm{T}$ transition}

While the spectral changes encompassing the L/T transition are driven largely by condensate depletion, what serves as the trigger? Temperature is clearly a factor, as analyses of color-magnitude diagrams suggest that most brown dwarfs transition from late- $\mathrm{L}$ to early-T over a narrow $T_{\text {ef } f}$ range (100-200 K) around $1200 \mathrm{~K}$ (Faherty et al. 2012).

1 This is the case for SDSS J0006-0852AB, where the lack of $\mathrm{H} \alpha$ emission in both SDSS J0006-0852A and its wide companion LP 704-48 indicate an age $\gtrsim 8 \mathrm{Gyr}$, consistent with the $\gtrsim 4 \mathrm{Gyr}$ age inferred from the analysis described above.

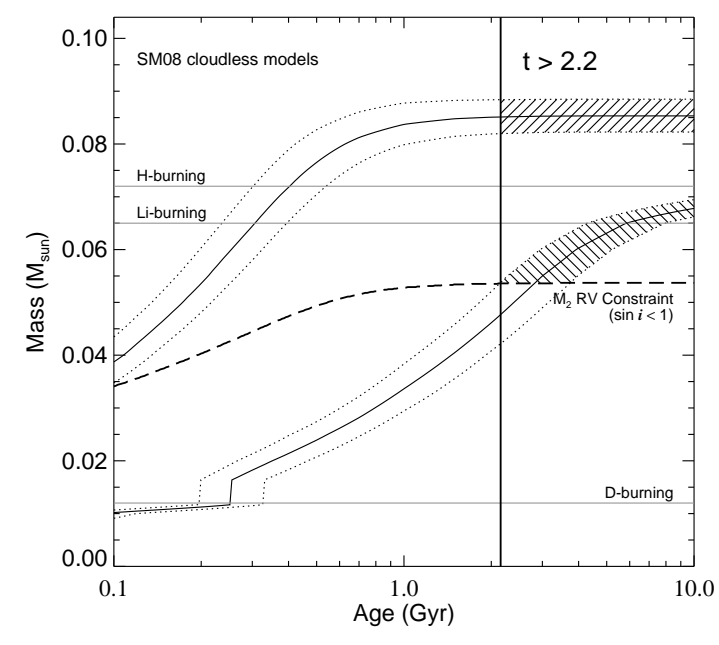

Fig. 2 Age and mass constraints for the tight spectral binary 2MASS J0320-0446AB, based on component spectral types (curved solid and dotted lines), RV orbit (dashed line) and evolutionary models from Saumon \& Marley (2008). This analysis constrains the primary mass to $0.085 \pm 0.025 \mathrm{M}_{\odot}$ and the age of the system to $>2 \mathrm{Gyr}$ (from Burgasser \& Blake 2009).

However, other parameters also play a role in triggering this transition. Young, low surface gravity brown dwarfs retain their clouds to lower $T_{\text {eff }} \mathrm{S}$ than field objects (Metchev \& Hillenbrand 2006; Saumon \& Marley 2008), as recently demonstrated by the unusually "dusty" exoplanets around HR 8799 (Currie et al. 2011) and 2MASS J1207-3932 (Barman et al. 2011). Theoretical work also supports a strong gravity dependence on the onset of the $\mathrm{L} / \mathrm{T}$ transition (Marley et al. 2012). Condensate production appears to be suppressed in metal-poor $\mathrm{L}$ dwarfs based on their enhanced $\mathrm{TiO}$ and metal-line absorption and extremely blue near-infrared colors (Burgasser et al. 2003; Gizis \& Harvin 2006); the transition to the as-yet undiscovered $\mathrm{T}$ subdwarf class may be far less dramatic as a result (Burrows et al. 2006). Finally, rotation may play a major role in driving surface inhomogeneities that disrupt cloud layers, although rotation measures (variability periods, $v \sin i$ ) are challenging for these intrinsically faint objects. Notably, the variable earlyT dwarfs SIMP J0136+0933 and 2MASS J2139+0220, both of which may have highly disrupted cloud decks, also have very different variability periods $(2.4 \mathrm{hr}$ and $7.8 \mathrm{hr}$, respectively). Additional examples are needed to quantify these effects.

\section{Future work}

The mechanism that drives the $\mathrm{L} / \mathrm{T}$ transition remains an outstanding problem in brown dwarf atmospheric science, and can be addressed by expanding our sample and characterization of single and multiple sources spanning this transition. Multi-wavelength variability studies hold the promise 
of confirming or refuting the patchy cloud model for this transition, but proper interpretation of observed lightcurves and the incidence of variability requires more detailed theoretical work on the $3 \mathrm{D}$ structure and temporal variation of rotating brown dwarf atmospheres. While 2D dynamic models have found that gravity waves can play an important role in stirring the photosphere (Freytag et al. 2010), a complete picture will require a 3D calculation that includes rotation effects. Given the advances in 3D modeling in interpreting brightness variations for transiting exoplanets (Knutson et al. 2007) and magnetic topologies of M dwarfs (Browning 2008), such work should be feasible.

Detailed characterization-resolved spectroscopy, parallaxes, and precise orbit determinations — of binaries straddling the $\mathrm{L} / \mathrm{T}$ transition can provide insight into the role that secondary parameters play in triggering the depletion of condensates; tight spectral binaries in particular can bring mass and age information to this problem. Finally, there is increasing evidence that sulfide cloud layers are emerging at the transition between the $\mathrm{T}$ and $\mathrm{Y}$ spectral classes (Morley et al. 2012). Lessons learned from mineral cloud behavior at the L/T transition will be essential for understanding the properties of these very cool atmospheres.

Acknowledgements. The author thanks Mark Marley, Jacqueline Radigan and Didier Saumon for helpful comments in the preparation of this article.

\section{References}

Ackerman, A. S. \& Marley, M. S. 2001, ApJ , 556, 872

Allard, F., Hauschildt, P. H., Alexander, D. R., et al. 2001, ApJ , 556,357

Artigau, É., Bouchard, S., Doyon, R., et al. 2009, ApJ , 701, 1534

Barman, T. S., Macintosh, B., Konopacky, Q. M., et al. 2011, ApJ 1, 735, L39

Basri, G. \& Reiners, A. 2006, AJ , 132, 663

Berger, E., Rutledge, R. E., Phan-Bao, N., et al. 2009, ApJ , 695, 310

Blake, C. H., Charbonneau, D., \& White, R. J. 2010, ApJ , 723, 684

Blake, C. H., Charbonneau, D., White, R. J., et al. 2008, ApJ L, 678, L125

Browning, M. K. 2008, ApJ , 676, 1262

Burgasser, A. J. 2007a, ApJ , 659, 655

Burgasser, A. J. 2007b, AJ , 134, 1330

Burgasser, A. J. \& Blake, C. H. 2009, AJ , 137, 4621

Burgasser, A. J., Cruz, K. L., Cushing, M., et al. 2010, ApJ , 710, 1142

Burgasser, A. J., Geballe, T. R., Leggett, S. K., et al. 2006a, ApJ , 637,1067

Burgasser, A. J., Kirkpatrick, J. D., Burrows, A., et al. 2003, ApJ , 592,1186

Burgasser, A. J., Kirkpatrick, J. D., Cruz, K. L., et al. 2006b, ApJ S, 166, 585

Burgasser, A. J., Liu, M. C., Ireland, M. J., et al. 2008a, ApJ , 681, 579

Burgasser, A. J., Looper, D. L., Kirkpatrick, J. D., et al. 2008b, ApJ , 674, 451

Burgasser, A. J., Luk, C., Dhital, S., et al. 2012, ApJ , 757, 110
Burgasser, A. J., Marley, M. S., Ackerman, A. S., et al. 2002, ApJ L, 571, L151

Burgasser, A. J., Sitarski, B. N., Gelino, C. R., et al. 2011, ApJ , 739,49

Burrows, A., Sudarsky, D., \& Hubeny, I. 2006, ApJ , 640, 1063

Cruz, K. L., Burgasser, A. J., Reid, I. N., et al. 2004, ApJ L, 604, L61

Currie, T., Burrows, A., Itoh, Y., et al. 2011, ApJ , 729, 128

Cushing, M. C., Marley, M. S., Saumon, D., et al. 2008, ApJ , 678, 1372

Cushing, M. C., Saumon, D., \& Marley, M. S. 2010, AJ , 140, 1428

Dupuy, T. J. \& Liu, M. C. 2012, ApJ S, 201, 19

Dupuy, T. J., Liu, M. C., \& Ireland, M. J. 2009, ApJ , 692, 729

Faherty, J. K., Burgasser, A. J., Walter, F. M., et al. 2012, ApJ , 752,56

Freytag, B., Allard, F., Ludwig, H., et al. 2010, A\&A, 513, A19

Geißler, K., Metchev, S., Kirkpatrick, J. D., et al. 2011, ApJ , 732, 56

Gelino, C. R. \& Burgasser, A. J. 2010, AJ , 140, 110

Gizis, J. E. \& Harvin, J. 2006, AJ , 132, 2372

Golimowski, D. A., Leggett, S. K., Marley, M. S., et al. 2004, AJ , 127,3516

Helling, C., Dehn, M., Woitke, P., \& Hauschildt, P. H. 2008, ApJ L, 675, L105

Joergens, V. 2008, A\&A, 492, 545

Khandrika, H., Burgasser, A. J., Melis, C., et al. 2013, AJ, submitted

Kirkpatrick, J. D., Looper, D. L., Burgasser, A. J., et al. 2010, ApJ S, 190, 100

Kirkpatrick, J. D., Reid, I. N., Liebert, J., et al. 1999, ApJ , 519, 802

Knapp, G. R., Leggett, S. K., Fan, X., et al. 2004, AJ , 127, 3553

Knutson, H. A., Charbonneau, D., Allen, L. E., et al. 2007, Nature, 447,183

Konopacky, Q. M., Ghez, A. M., Barman, T. S., et al. 2010, ApJ , 711,1087

Lane, C., Hallinan, G., Zavala, R. T., et al. 2007, ApJ L, 668, L163

Liu, M. C., Leggett, S. K., Golimowski, D. A., et al. 2006, ApJ , 647,1393

Looper, D. L., Gelino, C. R., Burgasser, A. J., et al. 2008a, ApJ , 685,1183

Looper, D. L., Kirkpatrick, J. D., Cutri, R. M., et al. 2008b, ApJ , 686,528

Marley, M. S., Saumon, D., Cushing, M., et al. 2012, ApJ , 754, 135

Marley, M. S., Saumon, D., \& Goldblatt, C. 2010, ApJ L, 723, L117

Marley, M. S., Seager, S., Saumon, D., et al. 2002, ApJ , 568, 335

Metchev, S. A. \& Hillenbrand, L. A. 2006, ApJ , 651, 1166

Metchev, S. A., Kirkpatrick, J. D., Berriman, G. B., et al. 2008, ApJ , 676, 1281

Morley, C. V., Fortney, J. J., Marley, M. S., et al. 2012, ApJ, 756, 172

Radigan, J., Jayawardhana, R., Lafrenière, D., et al. 2012, ApJ , 750,105

Route, M. \& Wolszczan, A. 2012, ApJ L, 747, L22

Sanchez-Lavega, A. 2001, A\&A, 377, 354

Saumon, D. \& Marley, M. S. 2008, ApJ , 689, 1327

Schubert, G. \& Zhang, K. 2000, in Astronomical Society of the Pacific Conference Series, Vol. 212, From Giant Planets to Cool Stars, ed. C. A. Griffith \& M. S. Marley, p. 210 
Tinney, C. G., Burgasser, A. J., \& Kirkpatrick, J. D. 2003, AJ , 126, 975

Vrba, F. J., Henden, A. A., Luginbuhl, C. B., et al. 2004, AJ , 127, 2948 